\title{
Diffeomorphisms and families of Fourier-Mukai transforms in mirror symmetry
}

\author{
Balázs Szendrői \\ Mathematics Institute, University of Warwick and Alfréd Rényi \\ Institute of Mathematics, Hungarian Academy of Sciences
}

\begin{abstract}
Assuming the standard framework of mirror symmetry, a conjecture is formulated describing how the diffeomorphism group of a Calabi-Yau manifold $Y$ should act by families of Fourier-Mukai transforms over the complex moduli space of the mirror $X$. The conjecture generalizes a proposal of Kontsevich relating monodromy transformations and selfequivalences. Supporting evidence is given in the case of elliptic curves, lattice-polarized K3 surfaces and Calabi-Yau threefolds. A relation to the global Torelli problem is discussed.
\end{abstract}

\section{Introduction}

Derived categories of coherent sheaves entered the mirror symmetry scene with the paper 16]. The mirror relationship as envisaged by Kontsevich is an equivalence of $\overline{A_{\infty}}$-categories built out of two manifolds $X$ and $Y$, both equipped with (complexified) symplectic forms and complex Calabi-Yau structures. One of these categories is $D^{b}\left(X_{t}\right)$, the bounded derived category of coherent sheaves on the complex manifold $X_{t}$, or a twisted version thereof. The other category is the derived Fukaya category $D^{b} \operatorname{Fuk}\left(Y, \omega_{0}\right)$ of the symplectic manifold $\left(Y, \omega_{0}\right)$, a category constructed from Lagrangian submanifolds and local systems on them, with morphism spaces given by Floer homology.

The origin of the equivalence of the two categories was not specified by Kontsevich. The picture was filled in by a proposal of Strominger, Yau and Zaslow [26], based on arguments coming from non-perturbative string theory. According to [26], mirror manifolds should be fibered into middle-dimensional real special Lagrangian tori over a common base space; moreover, the two fibrations should in a suitable sense be dual to each other. As it was subsequently realized, this should supply the equivalence of categories proposed by Kontsevich by an analytic form of the Fourier-Mukai transform, converting Lagrangian submanifolds equipped with a local system on one manifold into holomorphic bundles or more generally sheaves on the mirror; see e.g. [18].

The next question that arises is the origin of the torus fibrations; recently, some proposals have been put forward by Gross-Wilson 10] and KontsevichSoibelman [17] to the effect that these should arise from certain degenerations of the complex structure on the Calabi-Yau manifolds. These degenerations, to so-called maximally degenerate boundary points or complex cusps in the CalabiYau moduli space, have been known to play a major role in mirror symmetry 
since the heroic age [6; the ideas of [10] and 17] show their significance in the new order of things.

Many of the details of the torus fibrations, the definitions of the categories and their equivalence, and the relation to more traditional notions such as curvecounting generation functions and variations of Hodge structures are missing or at best conjectural. However, this should not necessarily prevent one from investigating some further issues such as symmetries on the two sides of the mirror map. The study of these issues was also initiated by Kontsevich. He pointed out that the categorical equivalence implies a correspondence between symplectomorphisms on a Calabi-Yau manifold and self-equivalences of the derived category of coherent sheaves of its mirror, and gave some explicit examples of this relationship. In particular, he related symplectomorphisms arising from certain monodromy transformations to certain relatively simple self-equivalences on the mirror side in the case of the quintic, and proposed some more general constructions of self-equivalences. These ideas were carried further by SeidelThomas [25] and in a different direction by Horja 11], 112].

A common feature of these works is that they present a static picture: the symplectic form and complex structure are frozen, and symmetries compared. The main contribution of the present work is that by investigating these notions in families, some additional insight can be gained.

The language of kernels (derived correspondences) is used to define a group that I term the categorical mapping group of a family of complex manifolds. The main conjecture of the paper states that for a pair $(X, Y)$ of mirror Calabi-Yau manifolds, there should be a homomorphism from the diffeomorphism group of $Y$ to the categorical mapping group of the total space of the complex moduli space of $X$. A weaker version of the conjecture concerns cohomology actions on the two sides of the mirror map.

I investigate the proposed conjecture for families of Calabi-Yau manifolds of dimension at most three. After a brief look at the elliptic curve case, where the relevant group action was already known to Mukai [, I consider the case of K3 surfaces. Derived categories of sheaves on K3s have only been studied in the projective case, to which I restrict; this can be done elegantly in families by considering lattice-polarized K3 surfaces, a notion due to Nikulin. The diffeomorphism group has to be restricted also; in effect, the lattice polarization partitions $H^{2}$ (generically) into algebraic and transcendental parts a priori, and the allowable diffeomorphisms are supposed to respect this choice. One further issue that arises is an analogue of a theorem of Donaldson on the complex side, regarding orientation on the cohomology of K3 surfaces. Unfortunately, I cannot prove the required statement at present; it is formulated as Conjecture 5.4. As-

\footnotetext{
${ }^{1}$ The reader will excuse me for a short historical digression. Mukai remarks in the introduction of 22 that the result that the derived category of an elliptic curve carries an action of $S L(2, \mathbb{Z})$ modulo the shift 'seems to be significant'. This must be one of the first explicit hints to mirror symmetry in the mathematics literature. Another early hint is discussed in [21, Section 8]: a picture of Mori about the cone of curves of an abelian surface closely resembles a picture of Mumford about the compactification of a Hilbert modular surface; these cones become duals under mirror symmetry. It is a curious fact that Mori drew his picture during the fall of 1979 which is exactly the time when 22] was submitted.
} 
suming this, Theorem 5.5 confirms that for any allowable diffeomorphism, there is a family of categorical equivalences with the correct cohomology action. As an illustration I investigate this correspondence in some detail for toric families.

In the threefold case, I only discuss examples of elements of the categorical mapping group, mostly coming from birational contractions on the threefolds. A typical case of Horja's work emerges in a novel fashion, showing that indeed, the present version of the conjecture is needed to get a full picture. To conclude, I point out a relation to (counterexamples to) the global Torelli problem.

The purpose of this paper is to discuss the proposed framework. Detailed proofs of the assertions, together with some further examples and applications, will be given elsewhere.

\section{Acknowledgments}

Conversations and correspondence with Tom Bridgeland, Andrei Căldăraru, Mark Gross, Paul Seidel and Richard Thomas during the course of this work were very helpful indeed. I thank the MPI for hospitality during a brief stay in September 2000, where some of these ideas took shape. I especially thank Yuri Manin for the warm welcome and stimulating conversations; he also provided the starting point for these ideas with questions in 20 .

\section{Basic definitions}

For the purposes of the paper, a Calabi-Yau manifold is complex manifold $X_{0}$ with holonomy exactly $S U(n)$, in particular trivial canonical bundle. I assume everywhere below that $n=\operatorname{dim}_{\mathbb{C}}\left(X_{0}\right) \leq 3$. The subscript means that a particular complex structure is chosen on the differentiable manifold $X$; sometimes I write $X_{0}$ as a pair $\left(X, I_{0}\right)$ to make the complex structure explicit in notation. $D^{b}\left(X_{0}\right)$ or $D^{b}\left(X, I_{0}\right)$ denote the bounded derived category of coherent sheaves on $X_{0}$. The Mukai map

$$
v: D^{b}\left(X_{0}\right) \rightarrow H^{*}\left(X_{0}, \mathbb{C}\right)
$$

is defined for $U \in D^{b}\left(X_{0}\right)$ by

$$
v(U)=\operatorname{ch}(U) \cup \sqrt{\operatorname{td}\left(X_{0}\right)} .
$$

The Mukai pairing on cohomology

$$
H^{*}(X, \mathbb{Z}) \times H^{*}(X, \mathbb{Z}) \rightarrow \mathbb{Z}+i \mathbb{Z}
$$

is defined by

$$
\left(\alpha_{0}+\ldots+\alpha_{2 n}\right) \cdot\left(\beta_{0}+\ldots+\beta_{2 n}\right)=(-1)^{n-1} \sum_{j=0}^{2 n} i^{j} \int_{Y} \alpha_{j} \cup \beta_{2 n-j}
$$


where $\alpha_{i}, \beta_{i} \in H^{i}(X, \mathbb{Z})$; this pairing extends linearly to rational and complex cohomology. Let $\tilde{L}=H^{*}(X, \mathbb{Z})$ be the $\mathbb{Z}$-module equipped with the Mukai pairing; a marked family is a smooth family $\pi: \mathcal{X} \rightarrow S$ with topological fibre $X$ and with a fixed isomorphism $\phi$ of the local system $\mathrm{R}^{*} \pi_{*} \mathbb{Z}$ with the constant local system $\tilde{L}$ on $S$ respecting pairings.

A kernel (derived correspondence) between smooth projective varieties $X_{i}$, $i=1,2$ is by definition an object $U \in D^{b}\left(X_{1} \times X_{2}\right)$. There is a composition product on kernels given for $U \in D^{b}\left(X_{1} \times X_{2}\right)$ and $V \in D^{b}\left(X_{2} \times X_{3}\right)$ by the standard formula

$$
U \circ V=\mathbf{R} p_{13 *}\left(\mathbf{L} p_{12}^{*}(U) \stackrel{\mathbf{L}}{\otimes} \mathbf{L} p_{23}^{*}(V)\right) \in D^{b}\left(X_{1} \times X_{3}\right) ;
$$

here $p_{i j}: X_{1} \times X_{2} \times X_{3} \rightarrow X_{i} \times X_{j}$ are the projection maps. A kernel $U \in$ $D^{b}\left(X_{1} \times X_{2}\right)$ is invertible, if there is a kernel $V \in D^{b}\left(X_{2} \times X_{1}\right)$ such that the products $U \circ V$ and $V \circ U$ are isomorphic in $D^{b}\left(X_{i} \times X_{i}\right)$ to $\mathcal{O}_{\Delta_{X_{i}}}$, the (complexes consisting of) the structure sheaves of the diagonals.

A kernel $U \in D^{b}\left(X_{1} \times X_{2}\right)$ defines a functor

$$
\Psi^{U}: D^{b}\left(X_{2}\right) \rightarrow D^{b}\left(X_{1}\right)
$$

by

$$
\Psi^{U}(-)=\mathbf{R} p_{1 *}\left(U \stackrel{\mathbf{L}}{\otimes} p_{2}^{*}(-)\right),
$$

If $U$ is invertible then $\Psi^{U}$ is a Fourier-Mukai functor, an equivalence of triangulated categories. An invertible kernel also induces an isomorphism

$$
\psi^{U}: H^{*}\left(X_{2}, \mathbb{Q}\right) \rightarrow H^{*}\left(X_{1}, \mathbb{Q}\right)
$$

on cohomology, defined by

$$
\psi^{U}(-)=p_{1 *}\left(v(U) \cup p_{2}^{*}(-)\right) .
$$

$\Psi^{U}$ and $\psi^{U}$ are compatible via the Mukai map $v$. For Calabi-Yau manifolds of dimension at most four, $\psi^{U}$ is an isometry with respect to the Mukai pairing.

Finally if $X_{0}$ is a complex manifold and $\alpha \in H^{2}\left(X_{0}, \mathcal{O}_{X_{0}}^{*}\right)$, then there is a notion of an $\alpha$-twisted sheaf on $X_{0}$. This can either be thought of as a sheaf living on the gerbe over $X_{0}$ defined by $\alpha$, or more explicitly as a collection of sheaves on an open cover where the gluing conditions are twisted by a Cech representative of $\alpha$.

\section{Mirror symmetry and diffeomorphisms}

The mirror symmetry story begins with the data $\left(Y, I_{0}, \omega_{0}\right)$, where $Y$ is a differentiable manifold, $I_{0}$ is a Calabi-Yau complex structure on $Y$, and $\omega_{0}$ is a Kähler form in this complex structure giving rise to a unique Ricci-flat metrid?. The mirror of $\left(Y, I_{0}, \omega_{0}\right)$ is conjecturally found using the following procedure.

\footnotetext{
${ }^{2}$ The $B$-field is discussed in Remark 2.4 below.
} 


\section{Procedure 2.1}

Step 1 Choose a degeneration $\mathcal{Y} \rightarrow \Delta^{*}$ of $Y_{0}$ over a punctured multi-disc $\Delta^{*}$ with mid-point $P \in \Delta$, which is a complex cusp (large complex structure limit point), an intersection of boundary divisors of the complex moduli space with prescribed Hodge theoretic behaviour [21].

Step 2 The choice of complex cusp $P$ induces a torus fibration on $Y_{0}$ via a degeneration process [10], 17]. Arrange this fibration so that its fibres are (special) Lagrangian with respect to the complex structure $I_{0}$ and the symplectic form $\omega_{0}$.

Step 3 Dualizing this fibration (Strominger-Yau-Zaslow [26]) should yield, after an appropriate compactification, a compact differentiable manifold $X$, together with an isomorphism

$$
\operatorname{mir}_{P}: H^{*}(Y, \mathbb{Q}) \rightarrow H^{*}(X, \mathbb{Q}),
$$

relating algebraic cohomology to transcendental cohomology, and compatible with filtrations coming from the Leray spectral sequence of the torus fibration on the transcendental part and degree on the algebraic part.

Step 4 The symplectic form $\omega_{0}$ and complex structure $I_{0}$ on $Y$ induce a Calabi-Yau structure $\left(X, I_{0}^{\prime}\right)$ and a symplectic form $\omega_{0}^{\prime}$ on the manifold $X$.

Step 5 According to Kontsevich' homological mirror symmetry conjecture [16] (cf. also Manin [20]), the statement that $\left(Y, I_{0}, \omega_{0}\right)$ and $\left(X, I_{0}^{\prime}, \omega_{0}^{\prime}\right)$ are mirror symmetric is expressed by a equivalence of categories determined by the torus fibration,

$$
\operatorname{Mir}_{P}: D^{b} \operatorname{Fuk}\left(Y, \omega_{0}\right) \longrightarrow D^{b}\left(X_{0}\right) .
$$

On the left hand side, $D^{b} \operatorname{Fuk}\left(Y, \omega_{0}\right)$ is the derived Fukaya category, which is (conjecturally) constructed from Lagrangian submanifolds and flat bundles on them. This equivalence is compatible with the cohomology isomorphism $\operatorname{mir}_{P}$ of Step 3.

My main interest in this paper lies in an understanding of the action of the diffeomorphism group $\operatorname{Diff}^{+}(Y)$ of $Y$ on the above data. A diffeomorphism $\gamma \in \operatorname{Diff}^{+}(Y)$ maps a triple $\left(Y, I_{0}, \omega_{0}\right)$ to a new triple $\left(Y, \gamma^{*} I_{0}, \gamma^{*} \omega_{0}\right)$ and in particular it defines a symplectomorphism $\left(Y, \gamma^{*} \omega_{0}\right) \cong\left(Y, \omega_{0}\right)$. Lifting $\gamma$ to a graded symplectomorphism [24] induces an equivalence

$$
\tilde{\gamma}: D^{b} \operatorname{Fuk}\left(Y, \gamma^{*} \omega_{0}\right) \stackrel{\sim}{\longrightarrow} D^{b} \operatorname{Fuk}\left(Y, \omega_{0}\right) \bmod [1]
$$

i.e. well-defined up to translation; this comes from the fact that the lifting of $\gamma$ to a graded symplectomorphism is only well-defined up to shift.

Perform Procedure 2.1 on the pair of Calabi-Yau triples $\left(Y, I_{0}, \omega_{0}\right)$ and $\left(Y, \gamma^{*} I_{0}, \gamma^{*} \omega_{0}\right)$ with respect to the same complex cusp $P$ to obtain mirrors $\left(X, I_{0}^{\prime}, \omega_{0}^{\prime}\right)$ and $\left(X, I_{0}^{\prime \prime}, \omega_{0}^{\prime \prime}\right)$. Then there is a diagram of categorical equivalences up to translation, where the bottom arrow is defined by the others: 


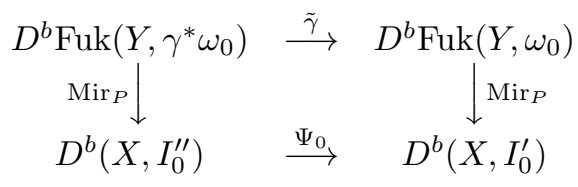

Further, the categorical equivalence $\Psi_{0}$ induces a cohomology isomorphism $\psi_{0}$ which must be compatible via the isomorphism $\operatorname{mir}_{P}$ with the action of $\gamma^{*}$ on $H^{*}(Y, \mathbb{Q})$.

To push this further, assume that $\left(I_{t}, \omega_{t}\right)$ is a family of complex structures and compatible symplectic forms on $Y$ deforming $\left(I_{0}, \omega_{0}\right)$. The diffeomorphism $\gamma$ induces a family of equivalences

$$
\tilde{\gamma}: D^{b} \operatorname{Fuk}\left(Y, \gamma^{*} \omega_{t}\right) \stackrel{\sim}{\longrightarrow} D^{b} \operatorname{Fuk}\left(Y, \omega_{t}\right) \bmod [1] .
$$

The mirrors of $\left(Y, I_{t}, \omega_{t}\right)$ and $\left(Y, \gamma^{*} I_{t}, \gamma^{*} \omega_{t}\right)$ with respect to the cusp $P$ are deformations $\left(X, I_{t}^{\prime}, \omega_{t}^{\prime}\right)$ and $\left(X, I_{t}^{\prime \prime}, \omega_{t}^{\prime \prime}\right)$ of the original $\left(X, I_{0}^{\prime}, \omega_{t}^{\prime}\right)$ and $\left(X, I_{0}^{\prime \prime}, \omega_{t}^{\prime \prime}\right)$. Consequently, there are induced equivalences

$$
\Psi_{t}: D^{b}\left(X, I_{t}^{\prime \prime}\right) \stackrel{\sim}{\longrightarrow} D^{b}\left(X, I_{t}^{\prime}\right) \bmod [1] .
$$

Slogan 2.2 Diffeomorphisms of the manifold $Y$ induce families of equivalences of derived categories up to translation over the complex moduli space of the mirror manifold $X$.

In the next Section, this slogan will be translated into a precise conjecture. The rest of the paper is providing evidence and examples. Before that however, there are two important points to clear up.

Remark 2.3 In order to be able to compare cohomology actions, it is necessary to work with marked moduli spaces of Calabi-Yau varieties in the procedure described above. This implies that the moduli spaces arising will typically be non-connected; this issue already arises for K3 surfaces. The procedure outlined above tacitly assumed that for $\gamma \in \operatorname{Diff}^{+}(Y)$, the complex structures $I_{0}$ and $\gamma^{*} I_{0}$ will be in the same connected component of the (marked) moduli space of complex structures on $Y$, and thus the same complex cusp $P$ may be used to form the mirror. In general, several components of the marked moduli space will contain complex structures related by diffeomorphisms. The way out is to pick a full set of components of the complex structure moduli space of $Y$ containing structures related by diffeomorphisms, and fix a complex cusp $P_{i}$ in each one of them in a compatible way so that the topological mirror $X$ and the cohomology map $\operatorname{mir}_{P}$ are the same for all cusps. Then the procedure described above is accurate. I tacitly assume this extension of the setup everywhere below. 
Remark 2.4 The above discussion ignores one crucial piece of data present in mirror symmetry, namely the $B$-field. In this remark I want to argue that, possibly under an extra assumption in the case of threefolds, I can consistently restrict to the case of vanishing $B$-field on the holomorphic side of mirror symmetry. The generalization of the present ideas to the case of arbitrary $B$-fields is left for future work.

Physical mirror symmetry deals with quadruples $\left(Y, I_{0}, \omega_{0}, B_{0}\right)$; here $B_{0} \in$ $H^{2}(Y, \mathbb{R} / \mathbb{Z})$ is the $B$-field on the Calabi-Yau manifold $\left(Y, I_{0}\right)$. The mirror of $\left(Y, I_{0}, \omega_{0}, B_{0}\right)$ is a quadruple $\left(X, I_{0}^{\prime}, \omega_{0}^{\prime}, B_{0}^{\prime}\right)$. According to a physics proposal originally formulated in the context of K-theory, for nonzero $B$-fields Kontsevich' homological mirror symmetry conjecture should take a form of an equivalence of categories

$$
\operatorname{Mir}_{P}: D^{b} \operatorname{Fuk}\left(Y, \omega_{0}, B_{0}\right) \longrightarrow D^{b}\left(X, I_{0}^{\prime}, B_{0}^{\prime}\right)
$$

see for example Kapustin-Orlov [13]. The category $D^{b} \operatorname{Fuk}\left(Y, \omega_{0}, B_{0}\right)$ on the left hand side should be a derived Fukaya-type category where the bundles on Lagrangian submanifolds are equipped with connections whose curvature is given by the restriction of $B$. For the current picture this is not a serious issue as diffeomorphisms should still give equivalences between these generalized derived Fukaya categories.

More importantly, the category on the right hand side should be a version of the derived category of coherent sheaves on $\left(X, I_{0}^{\prime}\right)$. More precisely, consider the natural map

$$
\delta: H^{2}\left(X_{0}, \mathbb{R} / \mathbb{Z}\right) \rightarrow H^{2}\left(X_{0}, \mathcal{O}_{X_{0}}^{*}\right) .
$$

Suppose first that the class $B$ has torsion image in $H^{2}\left(X_{0}, \mathcal{O}_{X_{0}}^{*}\right)$. In this case $D^{b}\left(X_{0}, B_{0}^{\prime}\right)$ should be the derived category of $\delta\left(B_{0}^{\prime}\right)$-twisted coherent sheaves $D^{b}\left(X_{0}, \delta\left(B_{0}^{\prime}\right)\right)$. If $\delta(B)$ is non-torsion, then $D^{b}\left(X_{0}, B_{0}^{\prime}\right)$ should be a suitable subcategory of a category of sheaves over a gerbe; for more discussion see [13, Remark 2.6].

Note that for elliptic curves, $H^{2}\left(X, \mathcal{O}_{X}^{*}\right)=0$ from the exponential sequence, so the derived category is not affected. However in higher dimensions, there are nontrivial twists.

Consider the case of $\mathrm{K} 3$ surfaces. The induced action of $\operatorname{Diff}^{+}(Y)$ on the space parameterizing quadruples $\left(X, I^{\prime}, \omega^{\prime}, B^{\prime}\right)$ is compatible via the cohomology isomorphism $\operatorname{mir}_{P}$ with the action on the rational cohomology $H^{*}(Y, \mathbb{Q})$. Moreover, by Proposition 5.2, the cohomology isomorphism $\operatorname{mir}_{P}$ is defined over $\mathbb{Z}$. So the induced action of $\operatorname{Diff}^{+}(Y)$ maps a zero $B^{\prime}$-field to a zero $B^{\prime}$-field.

In the case of Calabi-Yau threefolds, the isomorphism $\operatorname{mir}_{P}$ is not expected to be defined over $\mathbb{Z}$ but it is still defined over $\mathbb{Q}$ so it preserves torsion $B^{\prime}$ fields. On the other hand, in this case the torsion subgroup of $H^{2}\left(X, \mathcal{O}_{X}^{*}\right)$ is isomorphic to the torsion subgroup of $H^{3}(X, \mathbb{Z})$. Assuming $H^{3}(X, \mathbb{Z})_{\text {tors }}=0$, there are no possible torsion twists at all. As there is no known example of a smooth Calabi-Yau threefold with torsion in $H^{3}$, this restriction does not appear to be very serious.

I conclude therefore that for elliptic curves, K3 surfaces and Calabi-Yau threefolds under the assumption $H^{3}(X, \mathbb{Z})_{\text {tors }}=0$, it is legitimate to set $B^{\prime}=0$, 
as was effectively done in the above discussion.

\section{$3 \quad$ The categorical mapping group}

To translate Slogan 2.2 into a conjecture, I formalize the notion of a family of equivalences for complex structures on $X$. Assume that $\pi: \mathcal{X} \rightarrow \mathcal{D}$ is a family of complex projective varieties over a smooth complex base $\mathcal{D}$. Consider triples $(\phi, \alpha, U)$ where

- $S$ is an open subset of $\mathcal{D}$, the complement of a countable number of closed analytic submanifolds,

- $\phi: S \rightarrow \mathcal{D}$ is an analytic injection (not necessarily the identity), giving rise to the fibre product diagram

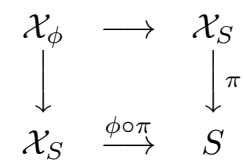

where $\pi: \mathcal{X}_{S} \rightarrow S$ is the restriction of the original family to $S$;

- $\alpha \in H^{2}\left(S, \mathcal{O}_{S}^{*}\right)$ is a twisting class on the base;

- $U$ is an object of the bounded derived category of quasi-coherent $p^{*} \alpha$ twisted sheaves on $\mathcal{X}_{\phi}$, whose derived restriction to the fibres of $p$ is isomorphic to a bounded complex of coherent sheaves on $X_{s} \times X_{\phi(s)}$; here $p: \mathcal{X}_{\phi} \rightarrow S$ is the natural map.

Let $M(\mathcal{X}, \mathcal{D})$ be the set of triples under obvious identifications, namely extension of $\phi$, and tensoring by a line bundle pulled back from the base $S . M(\mathcal{X}, \mathcal{D})$ can be given a monoid structure by a standard procedure generalizing the composition structure on kernels; the maps $\phi$ simply compose under the multiplication rule. The multiplication has a two-sided unit $\left(\operatorname{id}_{S}, 0, \mathcal{O}_{\Delta_{\mathcal{X}}}\right)$ where $\Delta_{\mathcal{X}} \subset \mathcal{X} \times_{S} \mathcal{X}$ is the relative diagonal.

Suppose further that $\mathcal{X} \rightarrow \mathcal{D}$ is a marked family with topological fibre $X$, and let $\tilde{L}_{\mathbb{Q}}=H^{*}(X, \mathbb{Q})$ be the rational cohomology of the fibres.

Definition 3.1 The categorical mapping group $G(\mathcal{X}, \mathcal{D})$ of the marked family $\pi: \mathcal{X} \rightarrow \mathcal{D}$ is the group of invertible elements $g=(\phi, \alpha, U)$ of the monoid $M(\mathcal{X}, \mathcal{D})$ satisfying the following cohomological condition $[$ : there should exist an isometry $\psi_{g} \in \operatorname{Aut}\left(\tilde{L}_{\mathbb{Q}}\right)$ and a commutative diagram of local systems on $S$

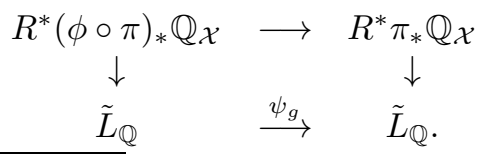

\footnotetext{
${ }^{3}$ Note that the cohomology condition is vacuous if $\mathcal{D}$ is connected.
} 
Here the vertical arrows come from the markings, and the top horizontal arrow is the isomorphism of local systems induced by the invertible relative kernel $U$.

The image $G^{c o h}(\mathcal{X}, \mathcal{D})$ of the resulting homomorphism

$$
\tilde{\psi}: G(\mathcal{X}, \mathcal{D}) \rightarrow \operatorname{Aut}\left(\tilde{L}_{\mathbb{Q}}\right) .
$$

is the cohomological mapping group of the family $\mathcal{X} \rightarrow \mathcal{D}$.

For an element $g=(\phi, \alpha, U) \in G(\mathcal{X}, \mathcal{D})$, derived restriction of $U$ to the fibre over $s \in S$ gives a well-defined untwisted object $U_{s}$ in $D^{b}\left(X_{s} \times X_{\phi(s)}\right)$. An easy base change argument shows that as $(\phi, \alpha, U)$ is invertible, $U_{s}$ is also invertible, and hence there is a Fourier-Mukai transform

$$
\Psi^{U_{s}}: D^{b}\left(X_{\phi(s)}\right) \rightarrow D^{b}\left(X_{s}\right)
$$

for every $s \in S$, with a well-defined cohomology action $\psi_{g}$ independent of $s \in S$.

The following main conjecture summarizes the discussion of the preceding sections.

Conjecture 3.2 Let $(\mathcal{Y} \rightarrow T, P)$ be a family of Calabi-Yau manifolds together with a choice of complex cusp $P \in \partial T$. Let $X$ be the topological mirror of $Y$ with respect to $P$; assume that $H^{3}(X, \mathbb{Z})_{\text {tors }}=0$. Let $\mathcal{X} \rightarrow \mathcal{M}_{X}$ be the marked Calabi-Yau moduli space (Teichmüller space) of $X$. Then there exists a homomorphism $\xi$ fitting into a commutative diagram

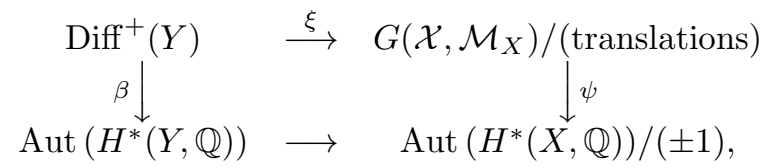

where

- translations mean relative translations over connected components on the base $\mathcal{M}_{X}$; moreover,

- $\beta$ is the natural action,

- $\psi$ is the map associating to an element $g$ of the categorical mapping group its cohomology action $\psi_{g}$ and

- the bottom horizontal arrow is the canonical map induced by the isomorphism $\operatorname{mir}_{P}$ of Procedure 2.1.

The diagram gives rise to an isomorphism of groups

$$
\bar{\xi}:\left\langle\operatorname{Difff}^{\mathrm{coh}}(Y),(-1)\right\rangle /( \pm 1) \longrightarrow G^{\mathrm{coh}, \text { filtr }}\left(\mathcal{X}, \mathcal{M}_{X}\right) /( \pm 1)
$$

where

- $\operatorname{Diff}{ }^{c o h}(Y)$ is the image of $\operatorname{Diff}^{+}(Y)$ in $\operatorname{Aut}\left(H^{*}(Y, \mathbb{Q})\right)$, and 
- $G^{\text {coh,filtr }}\left(\mathcal{X}, \mathcal{M}_{X}\right)$ is the subgroup of $G^{\mathrm{coh}}\left(\mathcal{X}, \mathcal{M}_{X}\right)$ consisting of elements whose associated cohomology action preserves the Leray filtration on the transcendental cohomology of $X$.

Remark 3.3 As discussed before, a diffeomorphism only acts on the derived Fukaya category up to translation. Hence in going from diffeomorphisms to their cohomology action, I have to take account of the cohomology action of translation [1] on the derived Fukaya category which is simply multiplication by $(-1)$. This is reflected on the left hand side of the cohomological form of the conjecture. On the right hand side, the cohomology actions of relevant families of Fourier-Mukai transforms must preserve the Leray filtration on the transcendental part of the cohomology of $X$; this is mirror to the statement that diffeomorphisms preserve the degree filtration on the algebraic cohomology of $Y$.

\section{Elliptic curves}

The diffeomorphism group Diff ${ }^{+}\left(T^{2}\right)$ of the two-torus acts via the standard $S L(2, \mathbb{Z})$-action on $H^{1}\left(T^{2}, \mathbb{Z}\right)$ and trivially on $H^{2}\left(T^{2}, \mathbb{R}\right)$. Thus, recalling the discussion of Section 2, Diff ${ }^{+}\left(T^{2}\right)$ should be realized by self-equivalences in the mirror family.

The elliptic curve is self-mirror. Choose cohomology classes dual to a collapsing circle fibre and a section of a circle fibration corresponding to the decomposition $T^{2}=(\mathbb{R} / \mathbb{Z})^{2}$ to fix an isomorphism

$$
\operatorname{mir}_{P}: H^{*}\left(T^{2}, \mathbb{Z}\right) \rightarrow H^{*}\left(T^{2}, \mathbb{Z}\right)
$$

interchanging even and odd cohomology. Let

$$
\mathcal{X}=\mathbb{C} \times \mathcal{H} /[(z, \tau) \sim(z+n+m \tau, \tau)], \quad m, n \in \mathbb{Z}
$$

be the universal family of marked elliptic curves together with the map

$$
\pi: \mathcal{X} \rightarrow \mathcal{H}
$$

to the marked moduli space, the upper half plane $\mathcal{H}$.

Theorem 4.1 There exists a group homomorphism

$$
\xi: \operatorname{Diff}^{+}\left(T^{2}\right) \rightarrow G(\mathcal{X}, \mathcal{H}) /(\text { translations })
$$

compatible with the isomorphism $\operatorname{mir}_{P}$ and descending to an isomorphism

$$
\bar{\xi}: \operatorname{PSL}(2, \mathbb{Z}) \stackrel{\sim}{\rightarrow} G^{\mathrm{coh}, \text { filtr }}(\mathcal{X}, \mathcal{H}) /( \pm 1) .
$$


Sketch Proof One only has to relativize the discussion of [25, Section 3d]; the $P S L(2, \mathbb{Z})$-action on the derived category of an elliptic curve was of course already known to Mukai [22]. There are no nontrivial twists over the base $\mathcal{H}$ and for all elements $(\phi, U) \in G(\mathcal{X}, \mathcal{H})$ in the image of $\xi$, the map $\phi$ is the identity.

\section{$5 \quad$ K3 surfaces}

\subsection{Lattice polarized mirror symmetry}

Let $M$ be an even non-degenerate sublattice of signature $(1, t)$ of the K3 lattice $L=H^{2}(X, \mathbb{Z})$. A marked ample $M$-polarized $K 3$ surface is a marked $K 3$ surface $\left(Y_{t}, \psi\right)$, which satisfies $\psi^{-1}(M) \subset \operatorname{Pic}(Y)$ and moreover $\psi^{-1}(\mathcal{C})$ contains an ample class for $\mathcal{C}$ a chosen chamber of $M_{\mathbb{R}}$; for the precise definition see [7]. For a pair $\left(Y_{t}, \psi\right)$, Hodge decomposition on the second cohomology gives the period point in

$$
\mathcal{D}_{M}=\left\{z \in \mathbb{P}\left(M_{\mathbb{C}}^{\perp}\right): z^{2}=0, z \cdot \bar{z}>0\right\} \backslash \bigcup_{\delta \in \Delta\left(M^{\perp}\right)}\langle\delta\rangle^{\perp}
$$

where $\Delta\left(M^{\perp}\right)$ is the set of vectors in $M^{\perp}$ of length -2 . The period domain $\mathcal{D}_{M}$ has two connected components.

Proposition 5.1 (河, Corollary 3.2]) The period map realizes $\mathcal{D}_{M}$ as the marked moduli space of marked ample $M$-polarized K3 surfaces; in particular, there exists a universal marked family $\pi: \mathcal{Y} \rightarrow \mathcal{D}_{M}$.

The mirror construction works best for a sublattice $M$ of $L$ with $M^{\perp}$ containing hyperbolic planes; I assume this from now on. The choice of a pair of vectors $\left\{f, f^{\prime}\right\} \subset M^{\perp}$ spanning a hyperbolic plane amounts to choosing a complex cusp $P \in \partial \mathcal{D}_{M}$. The choice of $\left\{f, f^{\prime}\right\}$ in $M^{\perp}$ also induces a splitting $M^{\perp}=\check{M} \perp\left\langle f, f^{\prime}\right\rangle$. This defines a new sublattice $\check{M}$ of $L$ of signature $(1,20-t)$ and gives rise to a family $\mathcal{X} \rightarrow \mathcal{D}_{\check{M}}$. This is the mirror family of $\mathcal{Y} \rightarrow \mathcal{D}_{M}$. The mirror construction, under the assumption on $M$, is an involution: starting with the pair $\left\{f, f^{\prime}\right\}$ thought of as defining a complex cusp in the boundary of $\mathcal{D}_{\check{M}}$, one recovers the original $M$-polarized family $\mathcal{Y} \rightarrow \mathcal{D}_{M}$. Monodromy and filtration considerations show

Proposition 5.2 The cohomology isometry

$$
\operatorname{mir}_{P}: H^{*}(Y, \mathbb{Q}) \rightarrow H^{*}(X, \mathbb{Q}),
$$

where $Y, X$ are thought of as fibres in $M$-, respectively $\check{M}$-polarized families, is given by

$$
\begin{gathered}
\operatorname{mir}_{P}\left(h_{0}\right)=f^{\prime}, \quad \operatorname{mir}_{P}\left(h_{4}\right)=-f, \quad \operatorname{mir}_{P}(f)=-h_{4}, \quad \operatorname{mir}_{P}\left(f^{\prime}\right)=h_{0}, \\
\operatorname{mir}_{P}(m)=m \text { for } m \in\left\langle h_{i}, f_{i}\right\rangle^{\perp} .
\end{gathered}
$$

\footnotetext{
${ }^{4}$ The choice in fact specifies a complex cusp in both components of $\mathcal{D}_{M}$; c.f. Remark 2.3 .
} 
In particular $\operatorname{mir}_{P}$ is defined over $\mathbb{Z}$.

In the framework of Procedure 2.1, I want to start with a general ample $M$-polarized $\mathrm{K} 3\left(Y, I_{0}, \omega_{0}\right)$ and I want to use the degeneration to the complex cusp $P \in \partial \mathcal{D}_{M}$ defined by $\left\{f, f^{\prime}\right\}$ in the $M$-polarized family $\mathcal{Y} \rightarrow \mathcal{D}_{M}$. The cohomology class of the the Kähler form has to be orthogonal to the period, so $\omega_{0}$ is restricted to live in the subspace $M_{\mathbb{R}} \subset H^{2}(Y, \mathbb{R})$. This condition has to be preserved throughout the whole procedure, hence the set of diffeomorphisms has to be restricted as well. Call $\gamma \in \operatorname{Diff}^{+}(Y) M$-allowable, if $\gamma^{*} \in O(L)$ satisfies $\gamma^{*}(M)=M$. Let $\operatorname{Diff}_{M}^{+}(Y)$ be the group of $M$-allowable diffeomorphisms of $Y$ and $\operatorname{Diff}_{M}^{\text {coh }}(Y)$ the image of $\operatorname{Diff}_{M}^{+}(Y)$ in the isometry group of $\tilde{L}$. Note that monodromy transformations around various rational boundary components of the marked moduli space $\mathcal{D}_{M}$ are certainly $M$-allowable.

\subsection{Action on cohomology}

Before I proceed further, let me recall

Theorem 5.3 (Donaldson [8) Let $\gamma \in \operatorname{Diff}^{+}(Y)$ be an orientation-preserving diffeomorphism of a K3 surface. Then the induced action on cohomology preserves the orientation of positive definite three-planes in $H^{2}(Y, \mathbb{R})$.

Comparing this theorem and the cohomology isomorphism $\operatorname{mir}_{P}$ given above leads to an important observation. Fix once and for all an orientation for the positive part of $H^{0}(X) \oplus H^{4}(X)$. Then for an algebraic K3 surface $X_{0}$ this, together with the real and imaginary parts of the period of $X_{0}$ and an ample class, gives an orientation to positive definite four-planes in the inner product space $H^{*}\left(X_{0}, \mathbb{R}\right)$, that I call for want of a better expression the canonical orientation. If the diffeomorphism group maps, at least on the level of cohomology, surjectively onto the cohomological mapping group, then Donaldson's theorem must have an analogue for Fourier-Mukai functors. I formulate this as

Conjecture 5.4 Let $\Psi: D^{b}\left(Z_{0}\right) \rightarrow D^{b}\left(X_{0}\right)$ be a Fourier-Mukai equivalence between derived categories of smooth projective K3 surfaces. Then the induced cohomology action $\psi: H^{*}\left(Z_{0}, \mathbb{Z}\right) \rightarrow H^{*}\left(X_{0}, \mathbb{Z}\right)$ preserves the canonical orientation of positive definite four-planes in cohomology.

\subsection{Cohomological realization of the main conjecture}

All the pieces are together; the following theorem confirms that Conjecture 3.2 can be realized on a cohomological level for mirror families of polarized K3 surfaces.

Theorem 5.5 Assume Conjecture 5.4. Let $P$ be a complex cusp in the boundary $\partial \mathcal{D}_{M}$ of the marked moduli space of the family of ample $M$-polarized K3

\footnotetext{
${ }^{5} \mathrm{~A}$ weaker form of this conjecture was proposed independently by Markman [19].
} 
surfaces. Let $\mathcal{X} \rightarrow \mathcal{D}_{\check{M}}$ be the mirror family. Then there exists an isomorphism

$$
\bar{\xi}:\left\langle\operatorname{Diff}_{M}^{\mathrm{coh}}(Y),(-1)\right\rangle /( \pm 1) \stackrel{\sim}{\longrightarrow} G^{\mathrm{coh}, \text { filtr }}\left(\mathcal{X}, \mathcal{D}_{\check{M}}\right) /( \pm 1)
$$

compatible with the isometry $\operatorname{mir}_{P}$.

Sketch Proof Let

$$
\sigma \in \operatorname{mir}_{P}\left\langle\operatorname{Diff}_{M}^{\mathrm{coh}}(Y),(-1)\right\rangle \operatorname{mir}_{P}^{-1} \subset O(\tilde{L})
$$

be an isometry of the Mukai lattice $\tilde{L}$. The task is to find an element $(\phi, \alpha, U) \in$ $G\left(\mathcal{X}, \mathcal{D}_{\check{M}}\right)$ with cohomology action $\sigma$.

Proposition 5.2 shows that $\sigma$ fixes $\check{M}^{\perp}=M \perp\left\langle f, f^{\prime}\right\rangle$. Therefore the map $\sigma^{-1}$ induces an automorphism

$$
\phi: \mathcal{D}_{\check{M}} \rightarrow \mathcal{D}_{\check{M}}
$$

mapping $[z] \in \mathcal{D}_{\check{M}}$ to $\left[\sigma_{\mathbb{C}}^{-1}(z)\right]$. The point about this map is that for $s \in \mathcal{D}_{\check{M}}, \sigma$ induces a Hodge isometry from $H^{*}\left(X_{\phi(s)}, \mathbb{Z}\right)$ to $H^{*}\left(X_{s}, \mathbb{Z}\right)$ that by Donaldson's Theorem 5.3 preserves the canonical orientation.

Work of Mukai 23] and global Torelli identifies $X_{\phi(s)}$ with a moduli space of sheaves on $X_{t}$ and this can be done in a relative way over an open subset $S \subset \mathcal{D}_{\check{M}}$ in the family $\mathcal{X} \rightarrow \mathcal{D}_{\check{M}}$. Some further arguments show that there exists a (suitably twisted) universal sheaf $U$ which defines an element of $G\left(\mathcal{X}, \mathcal{D}_{\check{M}}\right)$ with cohomology action $\sigma$.

\subsection{Toric families}

I want to give some examples of the correspondence between diffeomorphisms and families of Fourier-Mukai transformations in toric families. Let $\Delta \subset \mathbb{Z}^{3} \otimes \mathbb{R}$ be a three-dimensional reflexive lattice polyhedron [2]. It gives rise to a toric threefold $\mathbb{P}_{\Delta}$, which has a crepant toric resolution $\tilde{\mathbb{P}}_{\Delta} \rightarrow \mathbb{P}_{\Delta}$.

Let $\mathcal{X}_{\Delta} \rightarrow \mathcal{F}_{\Delta}$ be the family of anticanonical hypersurfaces $X_{t} \subset \tilde{\mathbb{P}}_{\Delta}$. For $t \in \mathcal{F}_{\Delta}, X_{t}$ is a smooth K3 surface and there is a restriction map Pic $\left(\tilde{\mathbb{P}}_{\Delta}\right) \rightarrow$ $\operatorname{Pic}\left(X_{t}\right)$ with image $M_{\Delta} \subset \operatorname{Pic}\left(X_{t}\right)$, the space of toric divisors on $X_{t}$. The equality $M_{\Delta}=\operatorname{Pic}\left(X_{t}\right)$ for general $t$ holds only under an extra condition, namely if for all one-dimensional faces $\Gamma$ of $\Delta$, neither $\Gamma$ nor its dual $\Gamma^{*} \subset$ $\Delta^{*}$ contain extra lattice points in their interior. If this condition fails, and it frequently does, then the Picard lattice of $X_{t}$ is not spanned by toric divisors.

Associated to the lattice $M_{\Delta}$ is a family of ample $M_{\Delta}$-polarized surfaces $\mathcal{X} \rightarrow \mathcal{D}_{M_{\Delta}} ; \mathcal{X}_{\Delta} \rightarrow \mathcal{F}_{\Delta}$ is a subfamily of this family. However, if the above condition fails, then the general ample $M_{\Delta}$-polarized K3 is not toric and $\mathcal{X}_{\Delta} \rightarrow$ $\mathcal{F}_{\Delta}$ is a proper subfamily of $\mathcal{X} \rightarrow \mathcal{D}_{M_{\Delta}}$.

Let $\Delta^{*}$ be the dual polyhedron of $\Delta$, and let $M_{\Delta^{*}}$ be the lattice of toric divisors of the Batyrev mirror family $\mathcal{Y}_{\Delta^{*}} \rightarrow \mathcal{F}_{\Delta^{*}}$. According to a conjecture 
of Dolgachev [7], there should exist a decomposition $M_{\Delta}^{\perp}=\left\langle f, f^{\prime}\right\rangle \perp \check{M}_{\Delta}$ as before, together with a primitive embedding

$$
i: M_{\Delta^{*}} \hookrightarrow \check{M}_{\Delta} .
$$

This conjecture is supported by numerical evidence and can be checked in several concrete cases [7, Section 8]; I assume that $\Delta$ is chosen so that the embedding exists. The embedding $i$ realizes inside the period domain

$$
\mathcal{D}_{M_{\Delta}} \subset \mathbb{P}\left(M_{\Delta}^{\perp}\right)_{\mathbb{C}}=\mathbb{P}\left(\check{M}_{\Delta} \perp\left\langle f, f^{\prime}\right\rangle\right)_{\mathbb{C}}
$$

the toric deformation space $\mathcal{F}_{\Delta}$ as an explicit subdomain

$$
\mathcal{F}_{\Delta}=\mathcal{D}_{M_{\Delta}} \cap \mathbb{P}\left(M_{\Delta^{*}} \perp\left\langle f, f^{\prime}\right\rangle\right)_{\mathbb{C}}
$$

(cf. [14, Section 4.3]).

The decomposition $M_{\Delta}^{\perp}=\left\langle f, f^{\prime}\right\rangle \perp \check{M}_{\Delta}$ gives the family $\mathcal{Y} \rightarrow \mathcal{D}_{\check{M}}$ of marked ample $\check{M}_{\Delta}$-polarized surfaces as the mirror family of $\mathcal{X} \rightarrow \mathcal{D}_{M_{\Delta}}$.

Turning to the specific issue of diffeomorphisms, Theorem 5.5 realizes, on the level of cohomology, $\check{M}_{\Delta}$-allowable diffeomorphisms on the family $\mathcal{X} \rightarrow \mathcal{D}_{M_{\Delta}}$ by Fourier-Mukai functors $\$$. The first examples are $\check{M}_{\Delta}$-allowable diffeomorphisms arising as monodromy transformations around boundary components meeting at the large complex structure limit point in the boundary of $\mathcal{D}_{\check{M}_{\Delta}}$.

Lemma 5.6 ([7, 6.2]) The monodromy transformations in boundary components meeting at the complex cusp $P \subset \partial \mathcal{D}_{\check{M}_{\Delta}}$ generate a group isomorphic to $M_{\Delta}$. The transformation corresponding to $d \in M_{\Delta}$ acts on the K3 lattice $L=H^{2}(Y, \mathbb{Z})$ by

$$
\begin{aligned}
& T_{d}(f)=f \\
& T_{d}\left(f^{\prime}\right)=f^{\prime}+d-\frac{d^{2}}{2} f \\
& T_{d}(m)=m-(d \cdot m) f \text { for } m \in\left\langle f, f^{\prime}\right\rangle^{\perp} .
\end{aligned}
$$

This action fixes the lattice $\check{M}_{\Delta} \perp\left\langle h_{0}, h_{4}\right\rangle$ pointwise, so a glance at the definition the map $\phi$ in the sketch proof of Theorem 5.5 shows that $\phi$ acts trivially on $\mathcal{D}_{M_{\Delta}}$. Indeed, by common wisdom, the monodromy transformation $T_{d}$ is mirrored by tensoring by the line bundle $\mathcal{O}_{X_{t}}(d)$ for $d \in M_{\Delta} \subset \operatorname{Pic}\left(X_{t}\right)$ and $t \in \mathcal{D}_{M_{\Delta}}$. In the relative framework, this is simply tensoring by a relative line bundle (twisted over the base if necessary).

However, as one moves away from these boundary components, diffeomorphisms with more complicated cohomology action crop up. In particular, there are diffeomorphisms $\gamma \in \operatorname{Diff}_{\check{M}_{\Delta}}^{+}(Y)$ whose associated cohomology action $\gamma^{*} \subset$ $O(L)$ fixes every element of $M_{\Delta^{*}}$ but does not fix the whole of $\check{M}_{\Delta}$ pointwise.

\footnotetext{
${ }^{6} \mathrm{~A}$ word of warning: the notation used here is related to that used in Theorem 5.5 by $M=\check{M}_{\Delta}$ and consequently $\check{M}=M_{\Delta}$.
} 
Hence these diffeomorphisms have mirrors with a nontrivial map $\phi: \mathcal{D}_{M_{\Delta}} \rightarrow$ $\mathcal{D}_{M_{\Delta}}$. This nontrivial map has a fixed locus which includes the toric locus $\mathcal{F}_{\Delta} \subset \mathcal{D}_{M_{\Delta}}$. The interpretation is that these diffeomorphisms are mirrored by families of Fourier-Mukai transforms acting as self-equivalences over the toric locus $\mathcal{F}_{\Delta}$, but acting by equivalences between derived categories of different K3s away from the toric locus. Compare Example 6.7.

\section{Threefolds}

\subsection{Examples of families of self-equivalences}

Example 6.1 Suppose that $\mathcal{X} \rightarrow \mathcal{M}_{X}$ is a marked family of Calabi-Yau threefolds. As in the previous section, some immediate examples of elements of $G\left(\mathcal{X}, \mathcal{M}_{X}\right)$ come from line bundles. Let $d \in H^{2}(X, \mathbb{Z})$; in the threefold case $d$ is automatically the first Chern class of a line bundle $\mathcal{O}_{X_{s}}(d) \in \operatorname{Pic}\left(X_{s}\right)$ on the fibres $X_{s}$ for $s \in \mathcal{M}_{X}$. There is a relative line bundle $\mathcal{O}_{\mathcal{X}}(d)$, possibly twisted over the base, which when thought of as a sheaf on the diagonal $\Delta_{\mathcal{X}} \subset \mathcal{X} \times \mathcal{M}_{X} \mathcal{X}$ gives an invertible relative kernel and so an element of $G\left(\mathcal{X}, \mathcal{M}_{X}\right)$ with $\phi$ the identity on $\mathcal{M}_{X}$. The fibrewise Fourier-Mukai transform is tensoring by $\mathcal{O}_{X_{s}}(d)$ in the derived category of $X_{s}$. These elements of $G\left(\mathcal{X}, \mathcal{M}_{X}\right)$ should again mirror monodromy transformations around components of the boundary of $\mathcal{M}_{X}$ meeting at the large structure limit point $P^{\prime} \in \delta \mathcal{M}_{X}$.

Example 6.2 A more interesting case where families of Fourier-Mukai selfequivalences arise is that of families of twist functors coming from contractions. Consider a Calabi-Yau threefold $X_{0}$ together with a flopping (or Type I) contraction $X_{0} \rightarrow \bar{X}_{0}$, a birational contraction with exceptional locus of dimension one. There is a diagram

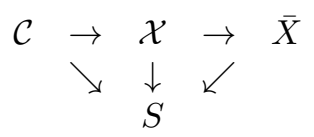

where $S \subset \mathcal{M}_{X}$ is a dense open subset in a connected component of the marked Calabi-Yau moduli space of $X_{0}$, and $\mathcal{C}$ is flat over $S$ intersecting every fibre in a chain of rational curves. The structure sheaf $\mathcal{O}_{\mathcal{C}}$ restricts to the fibre $X_{s}$ as $\mathcal{O}_{\mathcal{C}_{s}}$, a spherical sheaf in the sense of Seidel-Thomas [25]. This sheaf gives rise to a twist functor, a Fourier-Mukai self-equivalence of $D^{b}\left(X_{s}\right)$. There is an invertible relative kernel $U$, giving rise to an element $(\phi, 0, U)$ of $G\left(\mathcal{X}, \mathcal{M}_{X}\right)$ with $\phi$ the identical embedding of $S$ in $\mathcal{M}_{X}$ and restricting to the twisting kernel on $X_{s} \times X_{s}$ for $s \in S$.

Example 6.3 Suppose that a Calabi-Yau threefold $X_{0}$ has a Type II contraction, a birational morphism $X_{0} \rightarrow \bar{X}_{0}$ contracting an irreducible divisor $E_{0}$ to 
a point. $E_{0}$ is possibly non-normal but in any case it is a del Pezzo surface i.e. $\omega_{E_{0}}$ is ample. In this case, there is a diagram

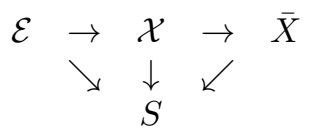

where $S \subset \mathcal{M}_{X}$ is dense in a connected component of the marked moduli space and the exceptional locus $\mathcal{E}$ is a flat family of surfaces over $S$ intersecting $X_{t}$ in a del Pezzo $E_{s}$.

The structure sheaf $\mathcal{O}_{E_{s}}$ of a del Pezzo surface is exceptional in the sense that there are no higher Ext's, so by [25, Proposition 3.15], its pushforward to $X_{s}$ is spherical. It defines a twist functor, a self-equivalence of the derived category of $X_{s}$. The construction works again over $S$; there is an invertible relative kernel defined by the family of surfaces $\mathcal{E}$ over $S$ which gives an element of $G\left(\mathcal{X}, \mathcal{M}_{X}\right)$ with $\phi$ still being the identity.

The elements of $G\left(\mathcal{X}, \mathcal{M}_{X}\right)$ given in Examples 6.2 6.3 should mirror monodromies around various other components of the boundary of moduli as discussed by Horja 11] and more recently by Aspinwall [1]. In particular, some of these transformations should mirror Dehn twists in Lagrangian three-spheres in the mirror, which are known to arise in degenerations to threefolds with double points; this was one of the starting points for Seidel and Thomas 25].

\subsection{An example with a nontrivial action on moduli}

In this section, I give an example of an element of the categorical mapping group with a nontrivial map $\phi$ on the base of moduli. The example comes from birational contractions on Calabi-Yau threefolds of Type III.

Example 6.4 Let $f_{0}: X_{0} \rightarrow \bar{X}_{0}$ be a birational contraction contracting a $\mathbb{P}^{1}$ bundle $E_{0}$ to a curve $C_{0}$ of genus at least two. On a dense open subset $S$ of the marked moduli space of $X$, the contraction extends to a diagram

$$
\begin{array}{cccc}
f: & \mathcal{X} & \rightarrow & \overline{\mathcal{X}} \\
& \downarrow & \swarrow & \\
S . & &
\end{array}
$$

The main new feature is that $f_{s}$ remains divisorial only for $s$ in a proper submanifold $S^{\text {div }} \subset S$. At a general point $s \in S$, the contraction $f_{s}: X_{s} \rightarrow \bar{X}_{s}$ is of flopping type, i.e. it contracts a codimension two locus; I assume for simplicity that it contracts disjoint $\mathbb{P}^{1} \mathrm{~s}$ to nodes.

By [15, Theorem 11.10], there is a flop in the family

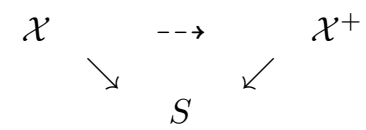


For $s \in S \backslash S^{\text {div }}$ where $f_{s}$ is of flopping type, $X_{s} \rightarrow X_{s}^{+}$is a classical flop. For $s \in S^{\text {div }}$, the map $X_{s} \rightarrow X_{s}^{+}$is the identity morphism.

The crucial point is that $\mathcal{X}^{+} \rightarrow S$ is the pullback of the original family $\mathcal{X} \rightarrow S$ under a nontrivial map $\phi: S \rightarrow S$. The fixed locus of $\phi$ is exactly $S^{\text {div }}$. There is an embedding

$$
\mathcal{X} \times_{\bar{X}} \mathcal{X}^{+} \hookrightarrow \mathcal{X} \times_{S} \mathcal{X}^{+}
$$

and by definition, the latter space is isomorphic to $\mathcal{X} \times_{\phi} \mathcal{X}$. Thus the structure sheaf of $\mathcal{X} \times{ }_{\bar{X}} \mathcal{X}^{+}$gives an element $U \in D^{b}\left(\mathcal{X} \times{ }_{\phi} \mathcal{X}\right)$.

Theorem 6.5 The triple $(\phi, 0, U)$ gives an element of $G\left(\mathcal{X}, \mathcal{M}_{X}\right)$.

Sketch Proof An easy argument shows that to prove that $(\phi, 0, U)$ is invertible, it is enough to show that the restriction is invertible on fibres. For $s \in S \backslash S^{\text {div }}, U$ restricts to $X_{s} \times X_{s}^{+}$as the structure sheaf of $X_{s} \times \bar{X}_{s} X_{s}^{+}$. This is the well-known kernel of Bondal and Orlov [3, Theorem 3.6], giving the isomorphism of derived categories under a classical flop.

For points $s \in S^{\text {div }}$, the restriction of $U$ to $X_{s} \times X_{s}$ is the kernel $\mathcal{O}_{X_{s}} \times_{\bar{X}_{s}} X_{s}$. The method of Bridgeland [4] can be used to show that this sheaf is a universal sheaf for a certain moduli problem on $X_{s}$ with fine moduli space $X_{s}$, and hence is an invertible kernel on $X_{s} \times X_{s}$.

Remark 6.6 The existence of the above kernel arising from Type III contractions was conjectured by Horja in 11], although he used a different expression [11, (4.35)]. In 12] he proves independently and by a different method that it is invertible. However, he does not discuss the relation to flops.

I illustrate the above discussion by a well-studied toric family.

Example 6.7 Start with a general degree eight hypersurface

$$
\bar{X}_{0} \subset \mathbb{P}^{4}\left[1^{2}, 2^{3}\right]
$$

in weighted projective four-space. Standard toric technology or a simple blowup give a crepant resolution $f_{0}: X_{0} \rightarrow \bar{X}_{0}$, which is a Type III contraction contracting a $\mathbb{P}^{1}$-bundle $E_{0}$ in $X_{0}$ to a genus 3 curve $C_{0}$ in $\bar{X}_{0}$. All toric deformations are resolutions of degree eight hypersurfaces in the weighted projective space; however, $h^{2,1}\left(X_{0}\right)=86$ whereas the octic has only 83 deformations, so some deformations of $X_{0}$ are missing.

To find the other deformations, embed $\mathbb{P}^{4}\left[1^{2}, 2^{3}\right]$ into $\mathbb{P}^{5}$ using $\mathcal{O}(2)$ as a hypersurface given by the equation $\left\{z_{1} z_{2}=z_{3}^{2}\right\}$. The variety $\bar{X}_{0}$ becomes a complete intersection

$$
\bar{X}_{0} \cong\left\{\begin{array}{l}
z_{1} z_{2}=z_{3}^{2} \\
f_{4}\left(z_{i}\right)=0
\end{array}\right\} \subset \mathbb{P}^{5}
$$


of a general quartic and a quadric of rank three in $\mathbb{P}^{5}$. The curve of singularities is along $\left\{z_{1}=z_{2}=z_{3}=0\right\}$. Here more deformations of $X_{0}$ are visible: I can deform either the quadric or the quartic. Deformations of $f_{4}$ contribute 83 moduli, which are exactly the toric deformations; there are 3 further deformation directions deforming the quadric to a quadric of rank fourl. The generic such deformation

$$
\bar{X}_{t}=\left\{\begin{array}{l}
z_{1} z_{2}=z_{3}^{2}-t^{2} z_{4}^{2} \\
f_{4}\left(z_{i}\right)=0
\end{array}\right\} \subset \mathbb{P}^{5}
$$

has four nodes at $\left\{z_{1}=z_{2}=z_{3}=z_{4}=0\right\}$ and a pair of small resolutions $X_{t}^{ \pm}$, which are deformations of $X_{0}$ :

$$
X_{t}^{ \pm}=\left\{\begin{array}{ll}
z_{1} u_{1} & =\left(z_{3} \pm t z_{4}\right) u_{2} \\
\left(z_{3} \mp t z_{4}\right) u_{1} & =z_{2} u_{2} \\
f_{4}\left(z_{i}\right) & =0
\end{array}\right\} \subset \mathbb{P}^{1} \times \mathbb{P}^{5}
$$

For $t \neq 0$, the birational map

$$
\psi_{t}: X_{t}^{+} \rightarrow X_{t}^{-}
$$

is a simple flop, flopping four copies of $\mathbb{P}^{1}$ over the four nodes of $\bar{X}_{t}$. By the main result of [27], for general $t$ the varieties $X_{t}^{ \pm}$are not isomorphic; what is more important from the point of view of the present discussion is that the flopped family explicitly appears as the original family under the base change $\phi: t \mapsto-t$.

In the connected component $\mathcal{X} \rightarrow \mathcal{M}_{X}$ of the Calabi-Yau moduli space of $X_{0}$, there is a sublocus $\mathcal{M}_{X}^{\text {div }} \subset \mathcal{M}_{X}$ where the contraction $f_{0}$ deforms as a divisorial contraction; this is also the fixed locus of the base change map $\phi$. On the other hand, as discussed above, $\mathcal{M}_{X}^{\text {div }}$ also equals $\mathcal{M}_{X}^{\text {toric }}$, the toric deformation space of $X_{0}$. The element $(\phi, 0, U)$ of $G\left(\mathcal{X}, \mathcal{M}_{X}\right)$ guaranteed by Theorem 6.5 acts as a self-equivalence on the toric deformations of $X_{0}$, but acts by FourierMukai transforms between different (generically non-isomorphic) deformations away from the toric locus. This is the same behaviour as that found at the end of Section 5.4 .

\subsection{Relation to the global Torelli problem}

The global Torelli theorem for K3 surfaces is an important tool in the proof of the cohomological form of Conjecture 3.2 for K3 surfaces. For Calabi-Yau threefolds, the situation appears to be exactly the opposite. As an invertible kernel induces a Hodge isometry on $H^{3}$, the following holds:

Proposition 6.8 Suppose that $\mathcal{X} \rightarrow \mathcal{M}_{X}$ is a component of the marked moduli space of a Calabi-Yau threefold. Assume that there is an element $(\phi, \alpha, U) \in$ $G\left(\mathcal{X}, \mathcal{M}_{X}\right)$ such that for general $s \in \mathcal{M}_{X}$, the varieties $X_{s}$ and $X_{\phi(s)}$ are not

\footnotetext{
${ }^{7}$ Deformations of $\bar{X}_{0}$ to non-singular complete intersections do not lift to deformations of the crepant resolution $X_{0}$.
} 
isomorphic. (In particular, $\phi$ is not the identity.) Then the global Torelli theorem fails for the family: the polarized (rational) Hodge structure on middle cohomology does not determine the general variety in the family up to isomorphism.

The counterexample to global Torelli given in 27] indeed comes from the nontrivial element of $G\left(\mathcal{X}, \mathcal{M}_{X}\right)$ of Theorem 6.5. Some conjectural examples of Calabi-Yau threefold families with a family of Fourier-Mukai transforms with nontrivial $\phi$ have been constructed by Andrei Căldăraru [5, Chapter 6]. These examples are of a very different flavour; they are elliptic fibrations without a section, the Fourier-Mukai transform coming from changing to a different elliptic fibration with the same Jacobian. The framework presented here suggests that counterexamples to global Torelli arising from families of Fourier-Mukai transforms should be rather more common than hitherto suspected. However, one may speculate that the isomorphism of the transcendental part of Hodge structures (in this case simply $H^{3}$ ) may imply the isomorphism of derived categories; this would be at the end of the day in complete agreement with the K3

case. At least for cohomology with integral coefficients, there appears to be no known counterexample to this speculation.

\section{References}

[1] P. S. Aspinwall, Some navigation rules for D-Brane monodromy, hepth/0102198.

[2] V.V. Batyrev, Dual polyhedra and mirror symmetry for Calabi-Yau hypersurfaces in toric varieties, J. Alg. Geom. 3 (1994) 493-535.

[3] A. Bondal and D. Orlov, Semi-orthogonal decomposition for algebraic varieties, math.AG/9506012.

[4] T. Bridgeland, Flops and derived categories, math.AG/0009053.

[5] A. Căldăraru, Derived categories of twisted sheaves on Calabi-Yau threefolds, Cornell Thesis 2000, available from http://www.math.umass.edu/ andreic.

[6] P. Candelas, X. de la Ossa, P.S. Green and L. Parkes, A pair of Calabi-Yau manifolds as an exactly soluble superconformal theory. Nuclear Phys. B 359 (1991), 21-74.

[7] I. Dolgachev, Mirror symmetry for lattice polarized K3 surfaces, Algebraic geometry, 4. J. Math. Sci. 81 (1996), 2599-2630.

[8] S. K. Donaldson, Polynomial invariants for smooth four-manifolds, Topology 29 (1990), 257-315. 
[9] M. Gross, Special Lagrangian fibrations I: Topology, Integrable systems and algebraic geometry (Kobe/Kyoto, 1997, eds. M.-H. Saito, Y. Shimizu and K. Ueno) 156-193, World Sci. Publishing, River Edge, NJ, 1998.

[10] M. Gross and P.M.H. Wilson, Large complex structure limits of K3 Surfaces, math.DG/0008018.

[11] P. Horja, Hypergeometric functions and mirror symmetry in toric varieties, math.AG/9912109.

[12] P. Horja, Derived category automorphisms from mirror symmetry, preprint February 2001.

[13] A. Kapustin and D. Orlov, Vertex algebras, mirror symmetry and D-branes: the case of complex tori, hep-th/0010293.

[14] M. Kobayashi, Duality of weights, mirror symmetry and Arnold's strange duality, alg-geom/9502004.

[15] J. Kollár and S. Mori, Classification of three-dimensional flips, J. Amer. Math. Soc. 5 (1992) 533-703.

[16] M. Kontsevich, Homological algebra of mirror symmetry, in: Proceedings of the International Congress of Mathematicians, (Zurich, 1994, ed. S.D. Chatterji), 120-139, Birkhauser, Basel, 1995.

[17] M. Kontsevich and Y. Soibelman, Homological mirror symmetry and torus fibrations, math.SG/0011041.

[18] N.C. Leung, S.-T. Yau and E. Zaslow, From special Lagrangian to Hermitian-Yang-Mills via Fourier-Mukai transform, math.DG/0005118.

[19] E. Markman, Letters to R. Thomas, January 1999.

[20] Yu. Manin, Moduli, motives, mirrors, ECM 2000 talk, math.AG/0005144.

[21] D.R. Morrison, Compactifications of moduli spaces inspired by mirror symmetry, Journées de Géométrie Algébrique d'Orsay (Orsay, 1992), Astérisque 218 (1993), 243-271.

[22] S. Mukai, Duality between $D(X)$ and $D(\hat{X})$ with its application to Picard sheaves, Nagoya Math. J. 81 (1981), 153-175.

[23] S. Mukai, On the moduli space of bundles on K3 surfaces. I, in: Vector bundles on algebraic varieties (Bombay, 1984), 341-413, Tata Inst., Bombay, 1987.

[24] P. Seidel, Graded Lagrangian submanifolds, Bull. Soc. Math. France 128 (2000), 103-149.

[25] P. Seidel and R. Thomas, Braid group actions on derived categories of coherent sheaves, math.AG/0001043. 
[26] A. Strominger, S.-T. Yau and E. Zaslow, Mirror symmetry is T-duality, Nuclear Phys. B 479 (1996), 243-259.

[27] B. Szendrői, Calabi-Yau threefolds with a curve of singularities and counterexamples to the Torelli problem, Int. J. Math. 11 (2000), 449-459.

Current address: Math. Inst., University of Warwick, Coventry CV4 7AL, UK

Email: balazs@maths.warwick.ac.uk 